\title{
Alignment and Integration of Lightweight Mirror Segments
}

\author{
Tyler Evans*a, Michael Biskach ${ }^{\mathrm{a}}$, Jim Mazzarella ${ }^{\mathrm{a}}$, Ryan McClelland ${ }^{\mathrm{a}}$, Timo Saha ${ }^{\mathrm{b}}$, Will Zhang ${ }^{\mathrm{b}}$, \\ Kai-Wing Chan ${ }^{\mathrm{C}}$ \\ aSGT, Inc, 7701 Greenbelt Road Suite 400, Greenbelt, MD 20770, USA; \\ bNASA Goddard Space Flight Center (GSFC), Greenbelt, MD USA 20771, USA; \\ 'Center for Research and Exploration in Space Science and Technology \& Center for Space \\ Science and Technology, University of Maryland, Baltimore County, Baltimore, MD 21250, USA;
}

\begin{abstract}
The optics for the International X-Ray Observatory (IXO) require alignment and integration of about fourteen thousand thin mirror segments to achieve the mission goal of 3.0 square meters of effective area at $1.25 \mathrm{keV}$ with an angular resolution of five arc-seconds. These mirror segments are $0.4 \mathrm{~mm}$ thick, and 200 to $400 \mathrm{~mm}$ in size, which makes it difficult not to impart distortion at the sub-arc-second level. This paper outlines the precise alignment, permanent bonding, and verification testing techniques developed at NASA's Goddard Space Flight Center (GSFC). Improvements in alignment include new hardware and automation software. Improvements in bonding include two module new simulators to bond mirrors into, a glass housing for proving single pair bonding, and a Kovar module for bonding multiple pairs of mirrors. Three separate bonding trials were x-ray tested producing results meeting the requirement of sub ten arc-second alignment. This paper will highlight these recent advances in alignment, testing, and bonding techniques and the exciting developments in thin $\mathrm{x}$-ray optic technology development.
\end{abstract}

Keywords: X-Ray Optics, International X-Ray Observatory, IXO Telescope, Alignment, Bonding

1.

INTRODUCTION

\subsection{Mission priorities}

$\mathrm{X}$-Ray astrophysics is an exciting field with the potential to discover things about matter under extreme conditions such as black holes, formation of structure such as dark matter, dark energy, and cosmic feedback, and the life cycles of matter and energy. The three main objectives when making optics for x-ray telescopes are angular resolution, effective area, and weight/cost. Since $x$-ray astrophysics involves looking at wavelengths from $\sim 100$ to 0.1 Angstroms, the photons would be absorbed into a mirror at a normal reflection angle. Therefore grazing incidence angles of the mirrors are required to be less than 2 degrees. This technology focuses on the Wolter-I type design utilizing a primary mirror and a secondary mirror to a prescribed focal distance. Because the grazing angle is so small, mirror segments must be nested extremely close to one another to meet the large effective area requirement. Thin segments pose their own challenges, as they can be easily distorted when trying to achieve such a high angular resolution. Previous X-ray missions have taken different approaches such as concentrating on optimizing angular resolution at the cost of effective area and weight, concentrating on angular resolution and effective area at the cost of weight, or focusing on weight at the cost of angular resolution and effective area. Table 1 below shows a comparison between three recent missions.

Table 1 - Comparing X-Ray Observatories ${ }^{3}$

\begin{tabular}{|c|c|c|c|c|c|}
\hline & $\begin{array}{c}\text { Angular } \\
\text { Resolution } \\
(\text { arcsec } \\
\text { HPV) }\end{array}$ & 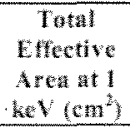 & $\begin{array}{c}\text { Total Mass } \\
\text { of } \\
\text { Telescope(s) } \\
\text { (kg) }\end{array}$ & $\begin{array}{l}\text { Protucturen } \\
\text { Cost }\end{array}$ & Note \\
\hline Cutand & 0.5 & 800 & -1800 & Wery High & 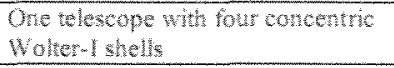 \\
\hline $\begin{array}{l}\text { XWhat } \\
\text { Newton }\end{array}$ & 5 & 4500 & -1600 & Moderate & 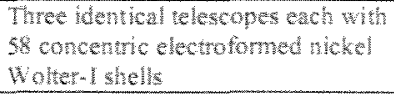 \\
\hline Suzark & 110 & 1200 & $-7 n$ & Low & 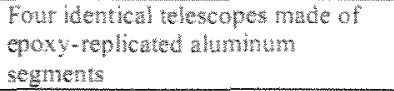 \\
\hline
\end{tabular}


*Tyler.Evans@nasa.gov; phone 1301 286-2677; ixo.gsfc.nasa.gov

The technology development outlined in this paper will try to optimize all three parameters by using thin glass mirrors to achieve good angular resolution, effective area, and low mass. Proving out the concept and procedures in the current technology development phase will hopefully enable streamlined mass production driving down the production cost.

\subsection{Mission design}

To accommodate all of the mirrors for the telescope, a modular design was conceived. The Flight Mirror Assembly ${ }^{2}$ (FMA) will support 60 modules arranged in three rings, 12 inner, 24 middle, and 24 outer. There will be 200 to 280 mirror segments per module for a total of about fourteen thousand mirror segments. The mirror segments being used are made by slumping flat glass onto polished mandrels ${ }^{5}$. The mirror segments are $200 \mathrm{~mm}$ long in the axial direction and have a circumferential span of up to $360 \mathrm{~mm}$. This makes each mirror about the size of a standard piece of 8.5 " by 11 " paper, and about four pieces of paper thick. Newer mirrors are being used with a span of 30 degrees instead of 50 degrees which are about half the size and better quality. Recent good results in the one mirror pair regime have shifted the goal towards proving out multiple pairs of mirrors into a module. The eventual telescope in a mission would be made up of thousands of mirrors. A modular approach would be used to populate an entire telescope with mirror segments consisting of hundreds of mirrors in each module, and rings of modules to fill out the telescope.

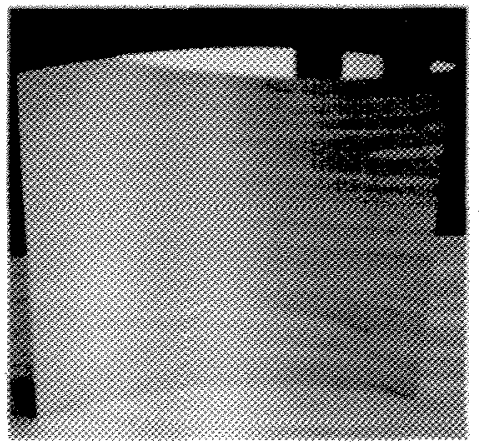

Mirror Segment

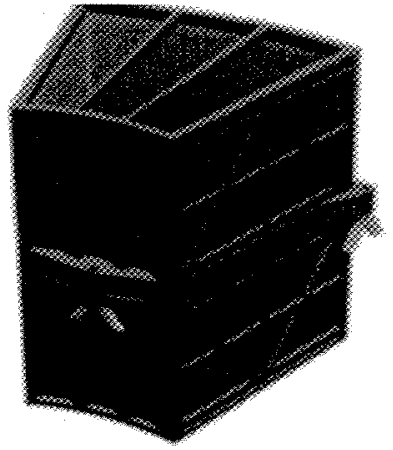

Mirror Module

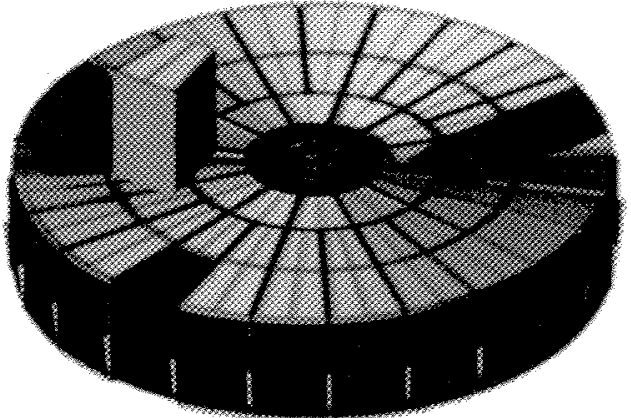

Telescope

Figure 1-Segment to Module to Telescope Comparison

Since the mirrors are not stacked on each other, but rather each independently attached to the housing, there is no stackup error, and procedurally, once three mirrors have been done, the process is just repeated for each additional pair.

2.

TEMPORARY BOND

\subsection{Suspension mount edge bond}

In June 2010, a new technique to temporarily hold the mirror to a strongback for alignment and bonding was developed. It is called "temporary edge-bonding". The method involved bonding three thin flexures to the top edge of the mirror and three screws to the bottom edge of the mirror. The idea behind this method was that the mirror is about 100 times stiffer in the vertical direction as opposed to the back surface that was previously used. This can be easily verified conceptually by folding a piece of paper in half at a 90 degree angle and standing it on its end. It is fairly stiff when pushing on the top of it, but if you push from the back, it deforms very easily. The same can be seen when building a house of cards, the cards are stiff on the axis parallel to their edges. 

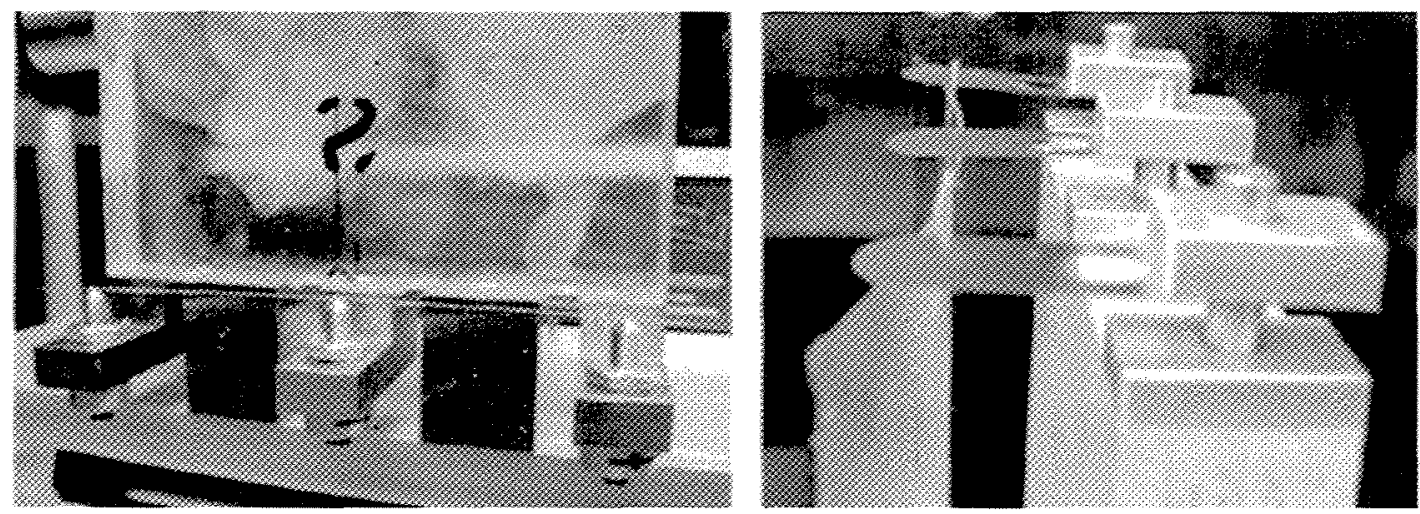

Figure 2 - Edge Temporary Bond Strongback Bottom Screws (left) and Top Flexures (right)

The interferometer data taken before and after mounting the mirror in this edge bond temporary mount was almost identical, to within the noise of the system. As shown in Figure 3, the left graph shows before temporary bonding, the right graph shows after temporary bonding. There are four main sections to the graph, the top pane shows the radius variation, the second pane shows the cone angle variation, the third pane shows the sag variation, and the bottom pane shows the mirror figure. The cone angle variation has the biggest variation on the mirror alignment performance. The mirror appears to get better after bonding, but it is within the noise of the system. Therefore, it was deemed a successful mount and adopted as the new way forward for temporarily mounting mirrors.
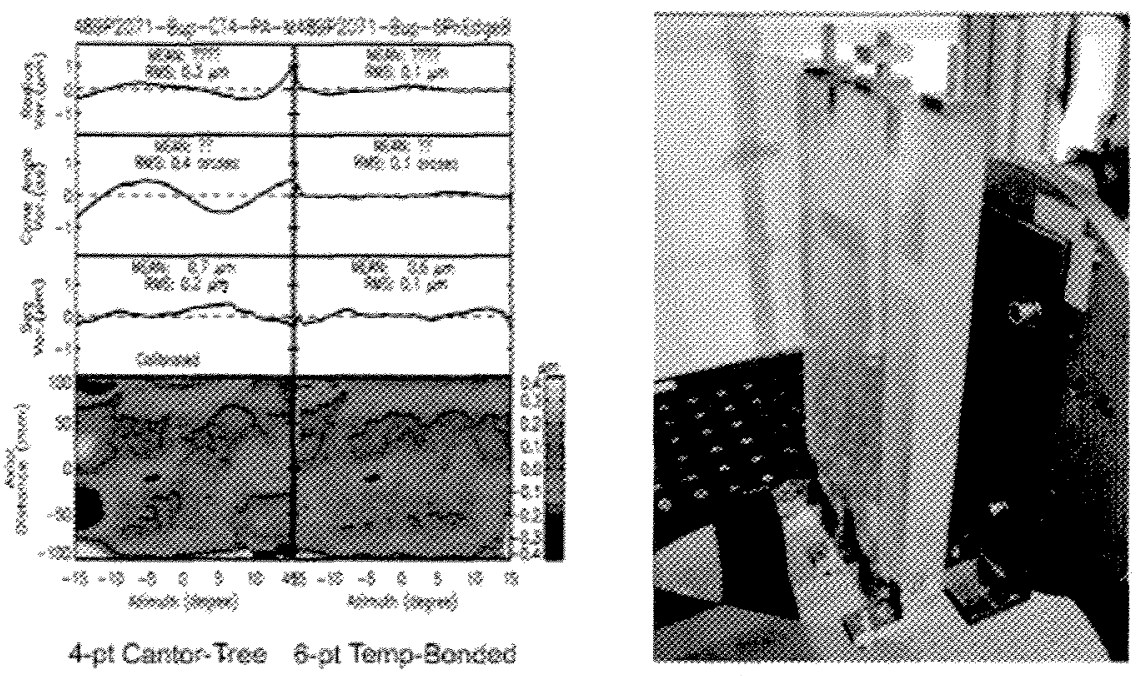

Figure 3 - Before and After Temporary Bonding Comparison (left) and Edge Bond Strongback on Hexapod (right)

It was later determined that only three points were required to form a mount closer to a kinematic mount and distort the mirror segment less. Therefore, two of the top flexures were removed, and one of the bottom screws was removed as can be seen in Figure 3. Therefore, the current version of temporary edge bonding involves a flexure on the top edge of the mirror segment, and two screws bonded to the bottom edge of the mirror.

The only trouble with this temporary bonding method was that it was fairly difficult to de-bond once the permanent bonds are attaching the mirror to the structure and the temporary mount has to be removed. The generic epoxy that was used to bond the mirror to the flexure and screws was dissolved using chemicals, but this was a tricky procedure to carry out without distorting the mirror.

\subsection{Kinematic Three Ball Mount (KB3)}

From the idea of holding the mirror at only three points, one on the top and two on the bottom edge spawned the idea of attaching small tooling balls directly to the mirror. Having these tooling balls essentially become part of the mirror allows for the mirror to be placed in three constraints, one cone, one slot, and one planar. This limits the number of degrees of freedom constrained and allows for less distortion of the mirror. It also reduces the temporary mounting time 
of a mirror from a day to an hour. This technique was named the Kinematic Three Ball Mount or KB3. A minimal structure holds the three capture devices that the balls of the mirror rest in. With just two KB3 mounts, and a supply of mirrors with balls pre-bonded onto them, alignment can be done very quickly by just swapping out mirrors in a process that takes less than a minute.
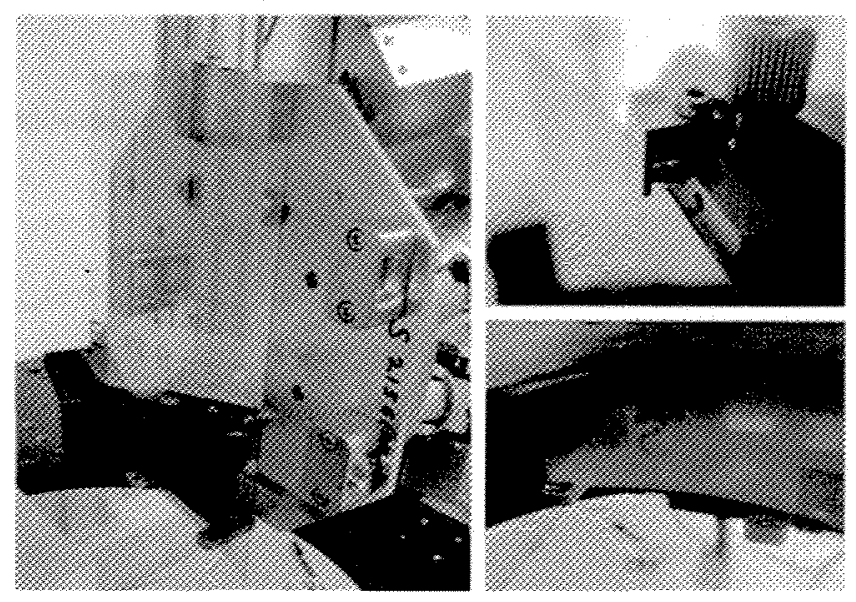

Figure 4-KB3 on Hexapod (left), Top Planar Constraint (upper right), Cone and Slot Constraint (lower right)

The KB3 mount has been a major breakthrough in speeding up turn-around time for having mirrors to work with. Because it can be used as a metrology mount as well as a temporary bond mount, it eliminates a step, saving time.

3.

MIRROR SEGMENT ALIGNMENT

\subsection{Mirror alignment}

Once the mirror is temporarily bonded, it can be tested for surface quality using interferometric metrology, and then put into proper alignment. Finite element modeling and test data confirm that small adjustments in re-orientation in the gravity field do not distort the mirror figure significantly. The alignment is done using a collimated beam of red light.

A six degree of freedom hexapod is used to align the strongback with the temporarily bonded mirror. The hexapod has a repeatability of $\pm 0.5 \mu \mathrm{m}$ in the linear $\mathrm{X}, \mathrm{Y}$, and $\mathrm{Z}$ directions (see Figure 5). The controller outputs the absolute position of the hexapod in $\mathrm{X}, \mathrm{Y}, \mathrm{Z}$ coordinates to $0.1 \mu \mathrm{m}$. The rotational position of the hexapod in $\mathrm{U}, \mathrm{V}$, and $\mathrm{W}$ coordinates is reported to $10^{-4}$ degrees. Knowing the absolute position of the mirror to this level of accuracy enables calculations to be performed to determine the necessary adjustments for optimizing the image.

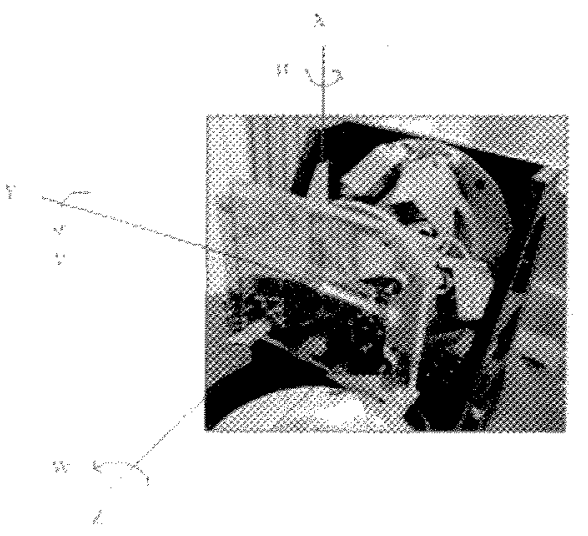


Figure 5-Hexapod coordinate system

The alignment is mainly adjusted by tilting the mirror in the $\mathrm{V}$ direction (pitch), and by rotating the mirror in the $\mathrm{W}$ direction (yaw). The final way to obtain a better image is to adjust the focal distance by moving the CCD camera at the focal plane. For data analysis purpòses in preliminary trials, it can be desirable to move the CCD camera to get better data to better understand the process. However, when multiple mirror pairs are permanently bonded, they must all focus at the same point since there will only be one detector.

There are three main focal distances that are used for the specific mirror segment being tested. The current mandrels for slumping glass segments are designed for the earlier mission specification of an 8.4 meter focal length. The focal lengths shown in table 2 are measured from the primary-secondary surface intersection point known as the PS point.

Table 2. Focal distances of various segments

\begin{tabular}{|c|c|}
\hline Type of Segment & Focal Distance (m) \\
\hline Primary & 16.804 \\
\hline Secondary & 5.654 \\
\hline Primary and Secondary & 8.400 \\
\hline
\end{tabular}

To achieve this long focal distance when the mirror is in a vertical position, a light source is positioned above the mirrors, shone downwards, and then bent 90 degrees using a 45 degree fold mirror so that it is parallel with the optical table. It is then bounced back and forth using flat fold mirrors to achieve the necessary focal length. The light source is a red beam with a wavelength of $633 \mathrm{~nm}$. Using visible light is a safer way to do testing than shorter wavelengths such as ultraviolet or $\mathrm{x}$-ray. Also, using visible light allows for the path of the light to be detected by the human eye by using white targets in order to find the image when large adjustments are made.

The mirror reflection starts as an arc shape (similar to the shadow of the curved mirror) which becomes smaller and smaller until it focuses to a small hourglass shape as shown in Figure 6 (also known as a rotated bow-tie). Past the focus, the arc becomes inverted, and grows in size. The focus location determines one component of the alignment. The location of the center of the hourglass itself determines the rest of the alignment. The location of the center of the hourglass is characterized by performing a Hartmann test.

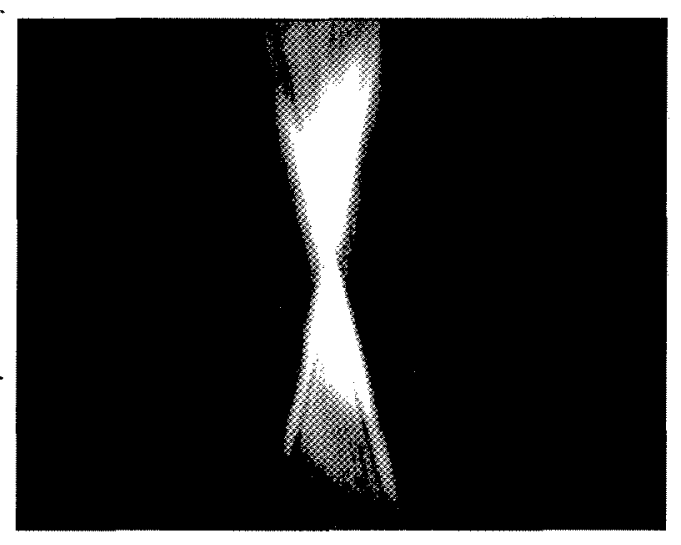

Figure 6-Image of mirror reflection at focus

Due to the light source generating a beam of light with a wavelength of about $633 \mathrm{~nm}$, there is a noticeable diffraction effect in the image. Because of the small cone angle of the primary mirror segment, this diffraction effect is three times larger for the primary mirror than for secondary mirror segments.

In order to achieve a good result, the mirror must be tilted at a very specific angle in which the light distribution at the focus is symmetrically distributed across the hourglass shape. A rough estimate of this symmetrical distribution of light can be done by simply looking at the image and correcting. For instance, as shown in Figure 6 , the light is slightly biased towards the right side of the image, which would be corrected by changing the yaw of the mirror. Biasing towards the 
top or bottom of the image can be adjusted by modifying the pitch angle. Fine tune adjustments are calculated using the analyzed data. Once a Hartmann test is complete, the general shape of the data set in addition to the magnitude of the errors can be used in conjunction with a set of equations to calculate the necessary adjustments needed for the optimal result. Because the relative position of the mirror between tests is known from the hexapod coordinates, it is possible to quantitatively calculate the adjustments.

\subsection{Mirror alignment Hartmann test}

A modified Hartmann test is used to test the alignment of the mirror. The test measures focusing of the mirror by measuring the light ray from sub-apertures of the mirror being tested. In the case of segmented cylinder-like mirror shells such as those of IXO, the simplest sub-aperture is an azimuthal slit. This simplifies the test significantly as the test is then a one-dimensional test.

To perform the test, a mask is used to cover the reflected light coming off of the mirror. Only specific slit locations are allowed to pass through the mask. The mask is then rotated to allow light from different strips of the mirror to be analyzed independently. When only a thin segment of the reflection arc is allowed to pass through the Hartmann mask, a line is displayed. When the lines formed by each stripe of the mirror are put together, they form the hourglass shape as shown in Figure 8. A new mask was designed to speed up the test and improve positional accuracy. Instead of the large $550 \mathrm{~mm}$ diameter circular mask that was used before with a two degree slit that was rotated two degrees at a time a new linear mask was designed. The new linear mask moves a radial slit across a section of an arc the same radius as the mirror segment being measured. The linear mask completes a Hartmann test in one minute whereas the circular mask used to take three minutes. Because as many as 30 tests can be run to iterate the alignment towards a solution for a single mirror, the time savings is substantial.
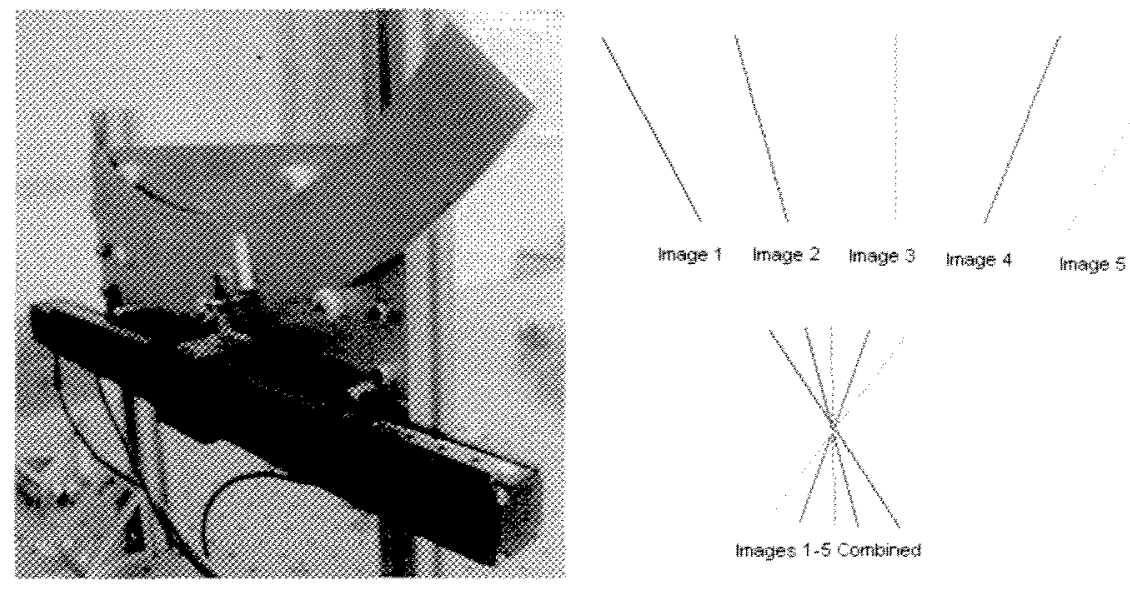

Figure 7 - Hartmann mask and test explanation (only five images shown to simplify diagram)

A CCD camera is used to capture an image of each line recording the brightness value of each pixel. The theoretical centroid of the brightness values should be in the center of the hourglass. Therefore the alignment error can be determined from the deviation between the centroids of each of the separate images. The final outcome of the test is a plot showing the deviation of each centroid location from the average location as shown in Figure 8. Motorized linear stages and a rotational motor have been utilized to automate this entire test. 


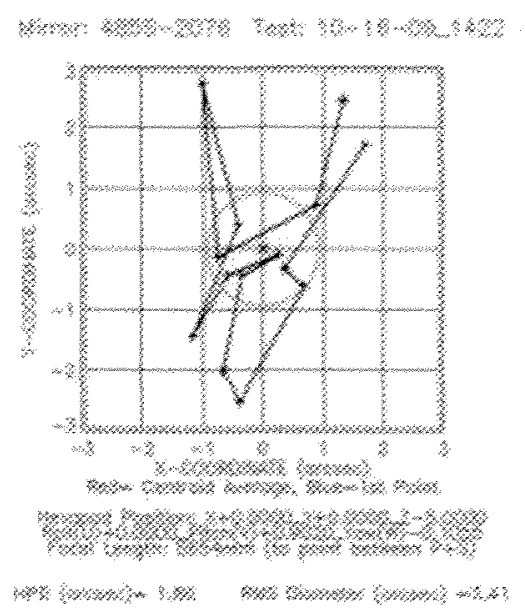

Figure 8 - Sample plot of centroids

The mirror segment alignment parameters are labeled on the graph to track settings used to achieve the image. This helps to understand what changed between trials to improve or degrade the image. The parameters are listed in five major categories. The mirror number is reported to show which mirror is being used. The test number reports the date and time the test was performed. The hexapod position shows the coordinates that the hexapod was programmed to in order to translate and tilt the mirror to the alignment used during the test. The focal length reports the distance between a fixed point $\mathrm{P}+\mathrm{S}$ and the CCD camera. The HPD and RMS ratings give a value of the spread of the centroids which is used to rate the mirror. The HPD rating of the mirror stands for "half power diameter". It is the diameter of the circle around the average centroid that would contain half of the points. It is signified by the magenta circle in Figure 8 . The blue cross signifies the first data point taken, which helps illustrate the shape of the mirror by tracking the individual points with the order they were taken in. The red $\mathrm{x}$ indicates the average of all the centroids.

\subsection{Hartmann test data analysis}

The data that is output after the Hartmann test is a set of images of single lines that when combined would form the "hourglass shape". Each image is analyzed independently to find the angle of a line that passes through the sliver of light. This line is represented by a dashed line in Figure 9.
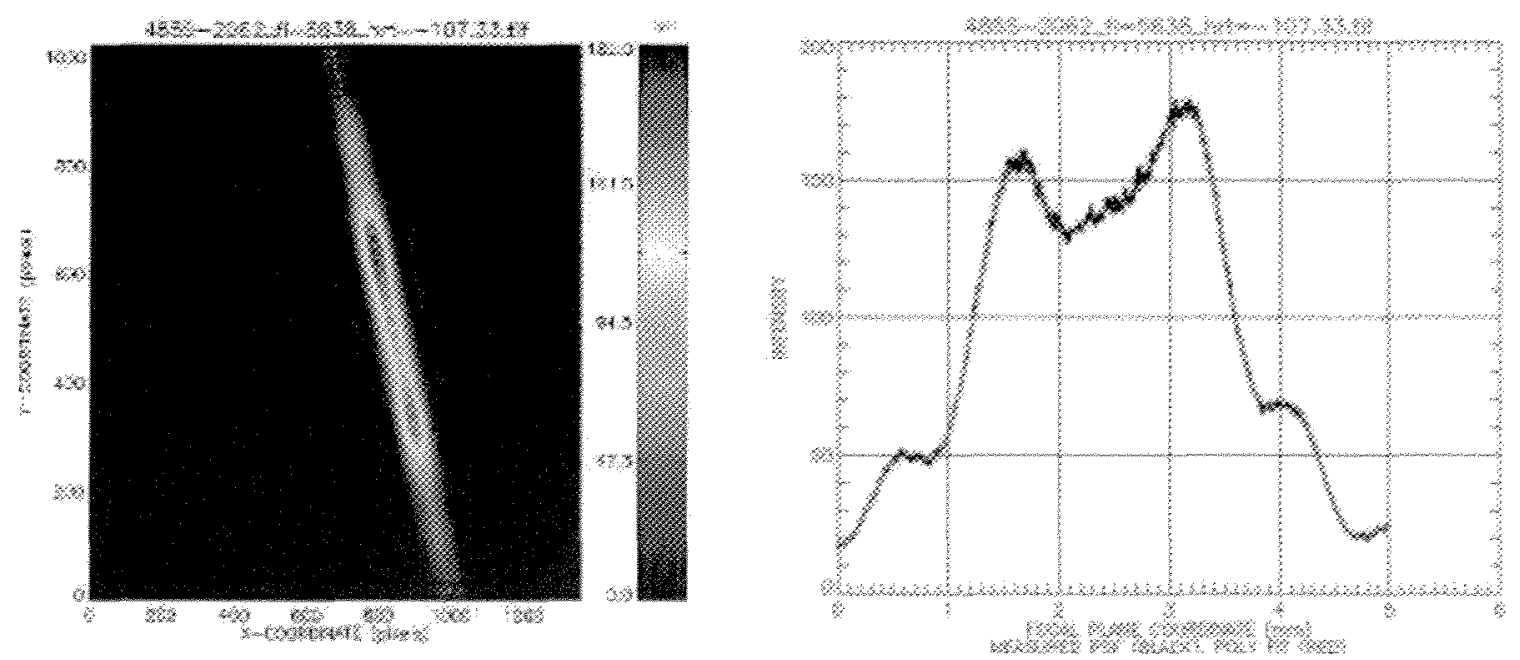

Figure 9 - Analyzed single image from Hartmann test (left), Light intensity curve along sliver of light (right)

Once this line has been found, the points along the line are analyzed to compare the brightness of each pixel. The light intensity as a function of focal plane coordinate is shown in Figure 9. The centroid of the area under this curve is 
calculated to determine image's centroid. This centroid represents where the center of the hourglass is for that specific image. By comparing the centroids of all of the images, the error rating of the mirror can be determined. Diffraction affects the result when using visible light, so the final test of the mirror alignment is done using $\mathrm{x}$-rays in a vacuum chamber. X-rays have a much shorter wavelength, and the diffraction effect is essentially negligible.

\subsection{Hartmann test automation}

Much of the Hartmann test has been automated to increase productivity and efficiency. The alignment of the mirror is controlled by a robotic hexapod through a Labview program. The Hartmann mask is moved on a slide using a motorized linear stage to isolate certain 2 degree sections of the mirror at a time for tests. The focal distance of the mirror is controlled by moving the camera on a motorized linear stage. The centroids are calculated in real-time as the Hartmann test is running and reported on the screen. The analysis software has been integrated into the Hartmann test control software so that analysis is automatically completed after the test is run. Considering two years ago the data had to be sent to an analyst to be computed, one year ago the data was analyzed in a separate program manually, and now the data is automatically analyzed as the test is running, the progression has been substantial. With this new tool it is much easier to run over night or weekend long tests of repeatability to see if the mirror alignment changes over time.

A new functionality has been added to the analysis program that not only plots a graph of the centroids, but also calculates the changes recommended to fix the pitch, yaw, focal distance, and primary to secondary mirror positions $\mathrm{x}$ and y positions. The user simply-has to enter in the correction value listed into the hexapod control and then take another Hartmann test. Through iteration, the alignment improves until the corrections recommended are within the noise of the test. Once extensive testing has been completed using this new automation program, the loop will be closed between the analysis corrections and the hexapod control so that the system automatically optimizes the alignment. Because the mirror is so close to the module walls however, any big movements would move the mirror into a wall breaking it. So precautions are being taken to verify the programming before final implementation.

\section{4.}

\section{PERMAMENT BONDING}

There is a variety of different housing equipment and permanent bonding methods that have been tried over the past year. They all consist of a Mirror Housing Simulator (MHS) which has the capability to bond one pair of mirrors or a Module Simulator (MS) which has the capability to bond multiple pairs of mirrors behind each other.

\subsection{Single pair 50 degree titanium MHS}

Once a mirror segment has been properly aligned, it was permanently bonded into a mock-up of the flight mirror module. For testing purposes, a Mirror Housing Simulator (MHS) was being used to provide bond locations similar to where they would be in the final module design. The MHS was capable of supporting three mirror pairs of different radii. The MHS was constructed of a Ti-15Mo alloy which fairly closely matches the coefficient of thermal expansion (CTE) of D263 glass mirror segments. 

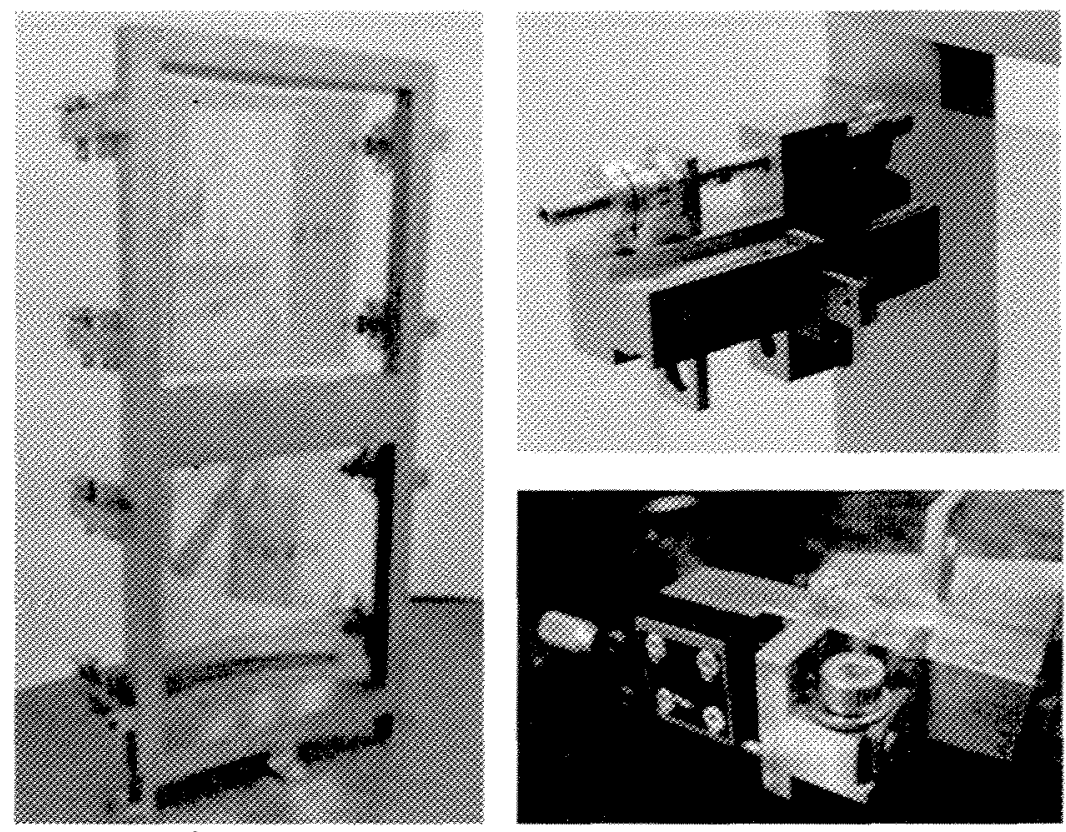

Figure 10 - MHS with two permanently bonded mirrors (left), tab alignment rail (top right), stage moving tab along rail (top right)

There are twelve rails, six on each side to hold the primary and secondary mirrors. For testing purposes only the rails at the four corners of each mirror were used as shown in Figure 10. Small flat tabs slide along the rails into position behind the mirror segment. A miniature stage was used to make the gap size between the tab and the mirror consistent to within a thousandth of an inch for all the tabs. Once in position, the tabs were secured to the rail using epoxy.

A closed loop system was designed for the bonding process with a laser displacement sensor (LDS) monitoring the position of the mirror to within 10 nanometers. The data from the LDS was fed into a program that controls the motion of a motorized linear stage called a nano-probe that moves the syringe in sub-micron steps to compensate for epoxy shrinkage and displacements caused by the epoxy making contact with the mirror and the tab. Since the final displacement requirement is less than 0.6 microns of movement, the process was automated so that it can be performed "hands off" without disturbing the MHS in between bonding tabs. The syringe and nano-probe were mounted to their own tower. Before any bonding occurs, 8 LDS are set up at each of the 4 bond points for both mirrors. The permanent bond hardware is shown in Figure 11. 


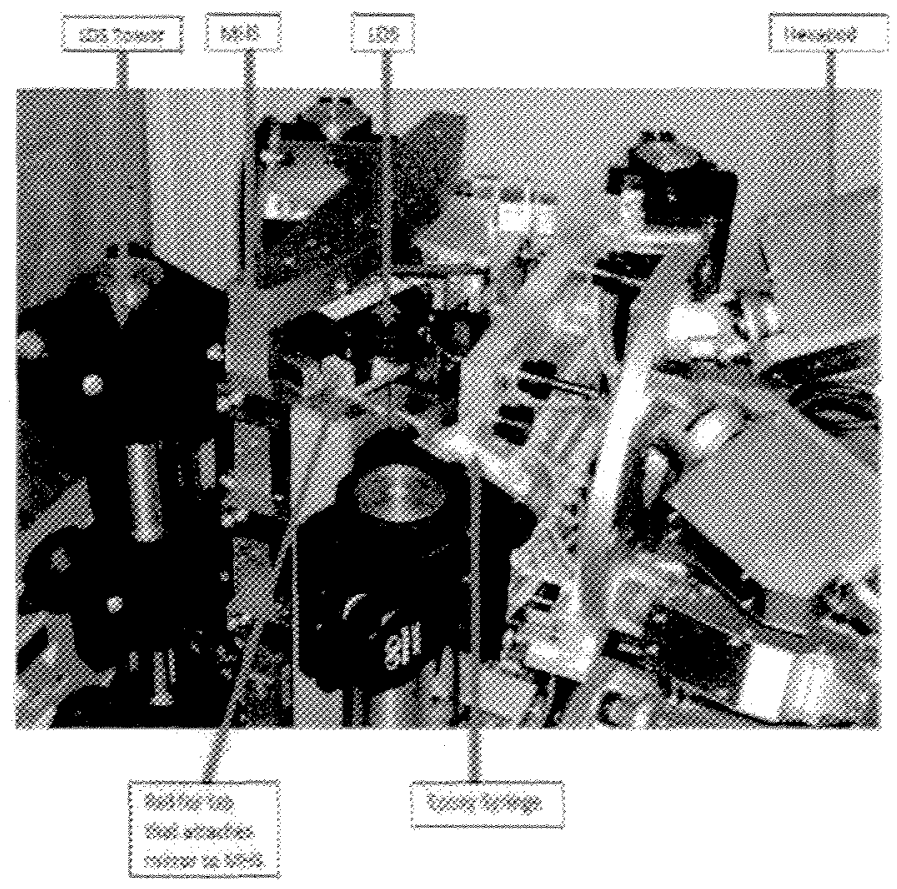

Figure 11 - Permanent bonding setup

Once the mirror has been bonded to all four tabs, the temporary bonds are broken by twisting the pins, and the strongback is removed. It has been demonstrated that breaking the temporary bonds does not damage the mirror.

\subsection{Single pair 30 degree Glass Mirror Housing Simulator (GMHS)}

To accommodate the new smaller 30 degree mirror segments a new housing module was constructed. This time, glass was chosen as a material since it would exactly match the Coefficient of Thermal Expansion (CTE) of the glass mirror segments, as opposed to the titanium alloy used previously which was close, but not close enough. The Glass Mirror Housing Simulator (GMHS) consists of a solid rectangular chunk of glass tall enough to accommodate a primary and secondary mirror. The glass housing has feet attached to the bottom of it to secure it to a table, and arms sticking off of the sides through which pins can be attached to the mirror.
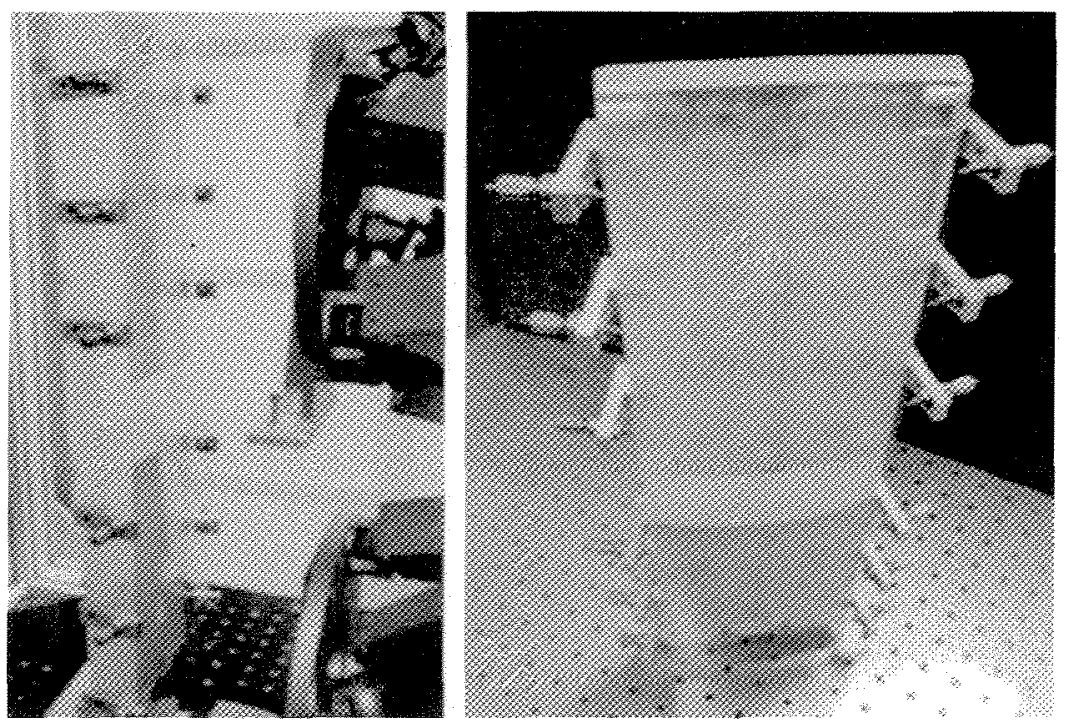

Figure 12-GMHS with Hexapods and Permanently Bonded Mirrors 
There are six arms for each mirror, three on each side for a total of twelve on the GMHS. The arms have slots through which a large fine pitch screw is secured The screw can move side to side and then be tightened down to reposition it based on the angular tilt and placement of the mirror. The screws are drilled out about 60 microns larger in diameter than the pins. Pins that are $1 \mathrm{~mm}$ in diameter are then inserted into the screw and pushed towards the mirror. The pins are attached to the mirror by using epoxy between the tip of the pin and the side edge of the mirror. While the epoxy is shrinking during cure, the pins are free to slide in or out of the screw, making up the difference in distance instead of pulling or pushing the mirror. Once the epoxy is cured, the pins are locked in place by wicking Loctite into the gap between the pin and the screw. Once the Loctite 222 cures and the pins are frozen in place, the mirror is fully secured in place. The temporary bonds are then broken by using an epoxy remover to dissolve the top flexure and two screws of the strongback to break the temporary bonds. Lastly, the mirror segment is left suspended in the GMHS, supported only by the six permanently bonded pins attached to the side edges.
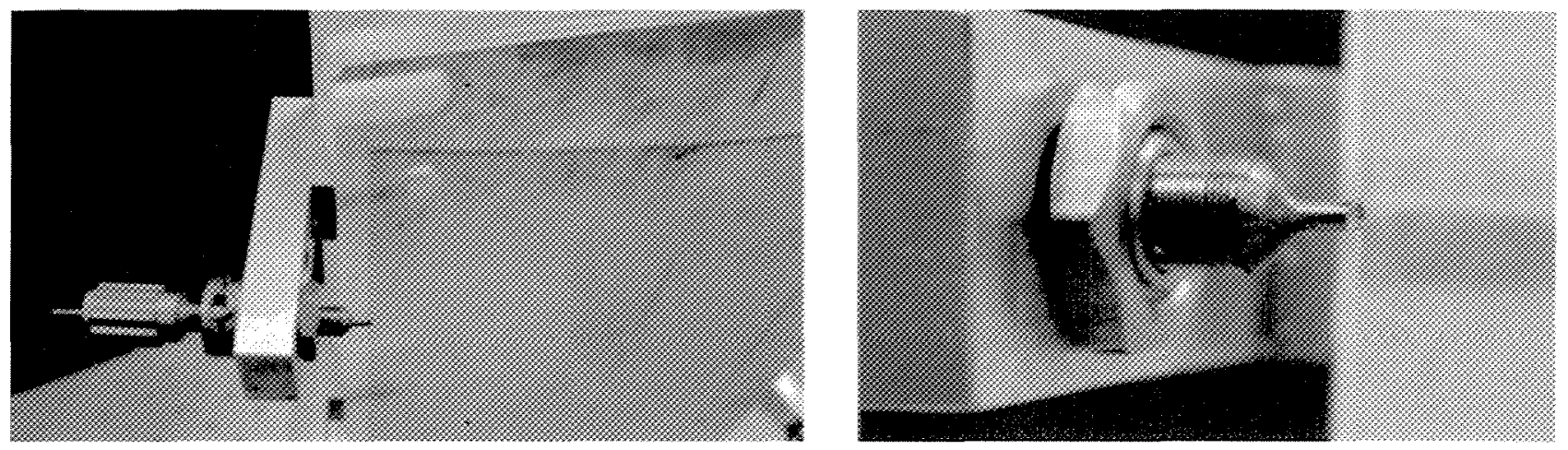

Figure 13 - GMHS Screw and Pin Setup for Permanently Bonding Mirror

\subsection{Multiple pair 30 degree Edge-bond Module Simulator}

A new Mirror Housing Simulator (MHS) was designed to accommodate multiple pairs of mirrors. The name changed from Housing Simulator to Module simulator since it is more like the flight module instead of just a simulator. The mirrors are not stacked or bonded to each other physically, but instead only attached to a common structure with this design concept. Because of this, once a procedure has been developed to integrate two to three mirrors, it can simply be repeated for any additional mirror segments needed to populate a module. The GMHS only had one set of screws and pins and therefore only supports one pair of mirrors. A move from a housing for a single pair of mirrors to a module for multiple pairs of mirrors was needed to prove out the technology. An Edge-Bond Module Simulator (EMS) was designed and built to accommodate this need.
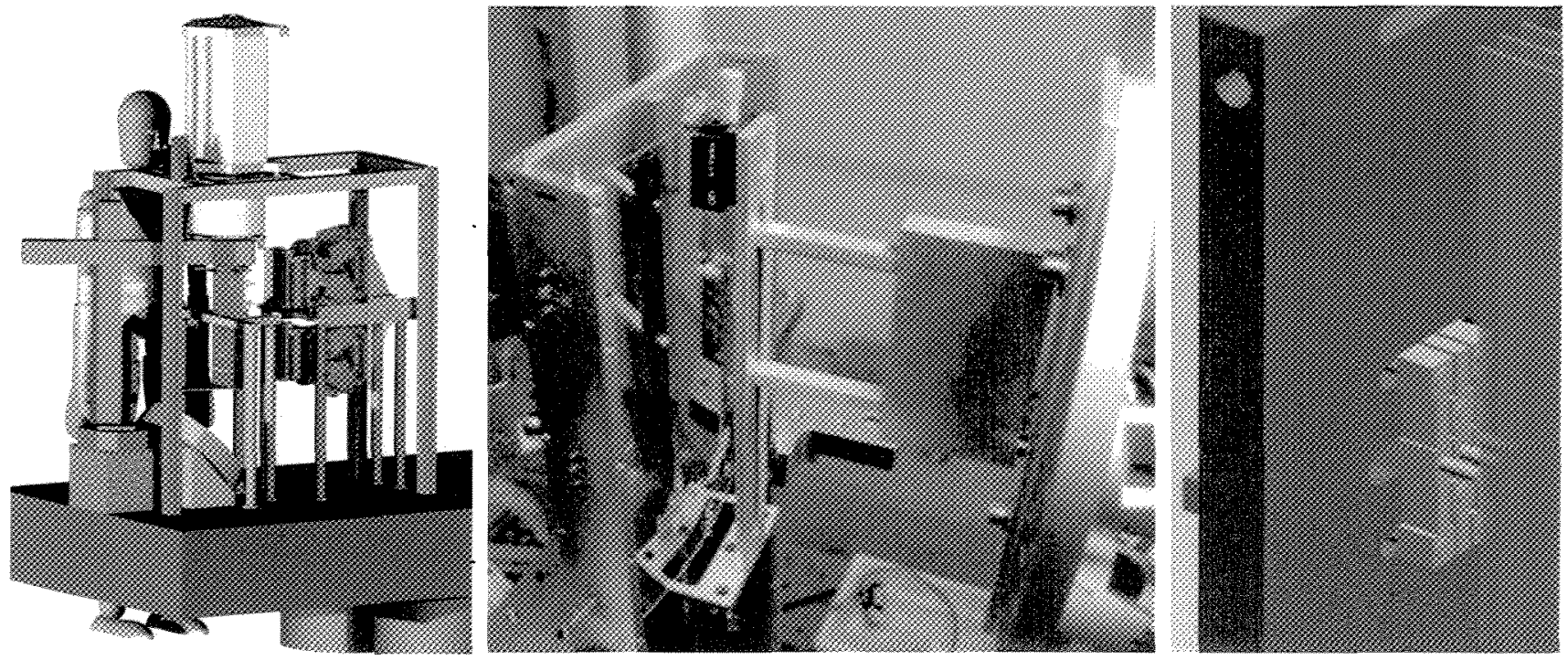

Figure 14 - VAF2 Setup (left), KB3 in EMS (middle), EMS Tab and Pin System with Three Mirrors (right) 
The design procedure for the EMS not only involved the module to house the mirrors, but also a re-design of the Vertical Alignment Facility (VAF). The new VAF2 design made changes to improve upon the original VAF based on lessons learned. The working height of the mirrors was lowered to a more convenient waist-high height as seen in Figure 14. Everything was designed as a complete system using Computer Aided Design (CAD) tools to fit-check components to existing hardware and structures. The structures holding the hexapods and the mirror module were separated for stability concerns so that hexapod movement would not affect the mirror alignment. Since some of the epoxy bonding steps require overnight cure cycles, long term stability is a major issue. An alignment laser guide was incorporated to calibrate multiple mirror pairs to a single focal point. Material choices of the support structures were all chosen carefully to match the optical bench and mirror substrates. The EMS structure was fabricated using Kovar, which closely matches the CTE of the glass segments, and can be much more precisely machined than glass. The large screws that were used to position the pins in the GMHS were too large to accommodate multiple mirror pairs separated by only $4 \mathrm{~mm}$. To solve this problem, new tabs were designed to hold the pins barely wider than the $1 \mathrm{~mm}$ diameter pins they support. The tabs are secured in place by bolting them to the EMS structure walls.

With the new EMS the procedure to bond in a single mirror was also changed slightly to improve the process. A camera was mounted to the end of a robotic arm to be able to provide a magnified view of the pin as it makes contact with the edge of the mirror. First, this helps remove the human head from being inches away from the mirror, putting out a significant amount of heat that was influencing the mirror. Second, it provides a much more stable view of the mirror output to a computer screen outside the bonding tent that shows a magnified view of the mirror and pin.
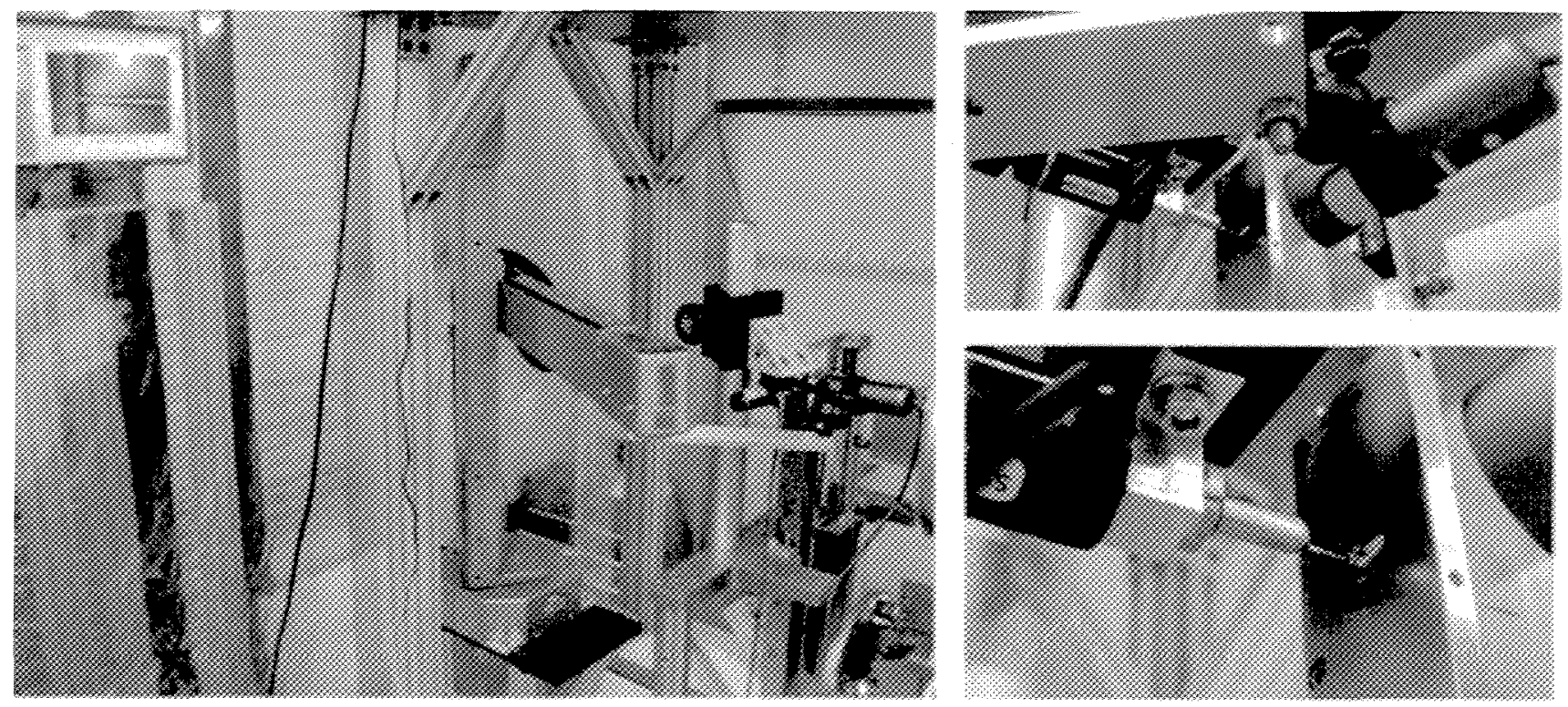

Figure 15 - Camera Setup and Nano-Actuator Pin Pusher

Since the pins being used are only one millimeter in diameter and the epoxy spot on the end of them is even smaller, it is hard to see details of when the pin epoxy exactly makes contact with the mirror with the naked eye. The robot can be easily commanded to move between the six pin locations to observe the pins as they are pushed to make contact with the edge of the mirror. A nano-actuator motorized probe was also introduced to control the movement of the pin. While watching the pin and mirror edge on the computer screen, the nano-actuator is extended in sub-millimeter steps to slowly move the pin until it contacts the mirror edge. The introduction of robotic and motorized components have helped turn the procedure into more of an engineered repeatable process instead of an art of doing it by hand. 

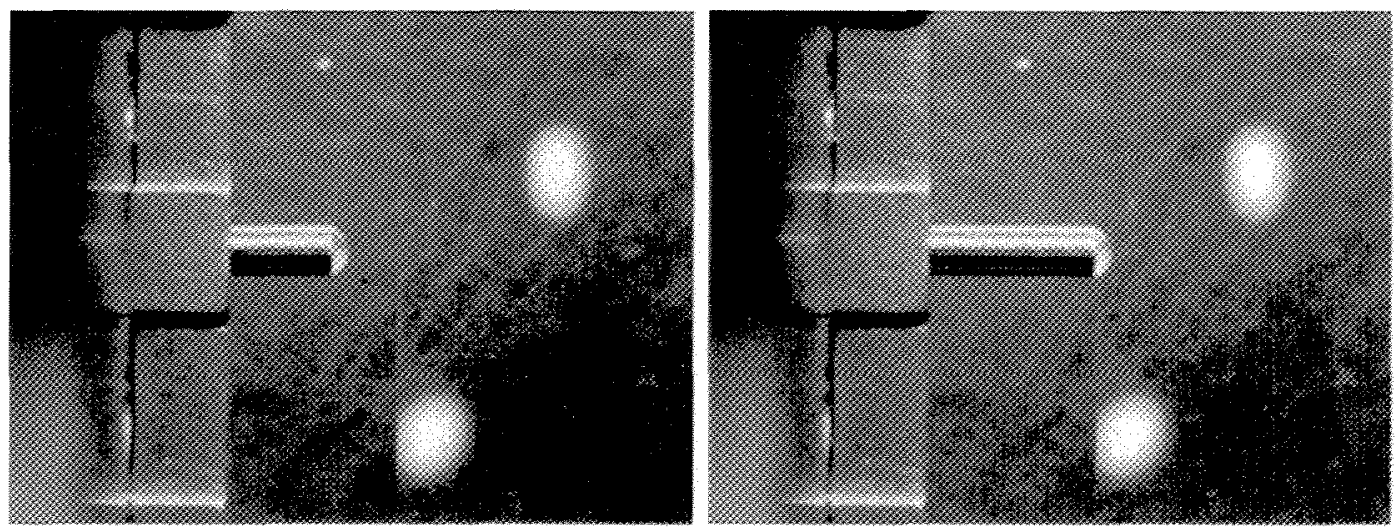

Figure 16 - Pin Before and After Being Pushed to Contact Mirror Edge

Once the pin makes contact with the mirror and the epoxy has cured, the pin is frozen into place by adding Loctite 222 into the pin/tab interface. This step is still done by hand as no better alternative has yet been implemented. Finally, the top constraint of the KB3 is removed, and the KB3 is then simply lowered by using the hexapod to move the bottom two constraints down away from the balls, releasing the mirror to be only supported by the six permanent pins.

5.

\section{RESULTS}

Several trials have been completed using multiple techniques, but three permanent bonding trials that were taken all the way through the process and then $\mathrm{x}$-ray tested will be highlighted.

\subsection{Titanium 50 degree MHS September permanent bonding and x-ray test}

The mirror pair 489C-2058 was bonded in September 2010. The overall result was the best ever achieved by the project, at the time and the mirror were taken to $\mathrm{x}$-ray testing for a more comprehensive analysis. The best alignment achieved ever by the project was achieved by these two mirrors on the hexapods with a result of 1.1 arc-seconds HPD, 1.9 arcseconds RMS diameter. The mirrors changed slightly after that result, and were at 4.0 arc-seconds RMS diameter at the start of permanent bonding. After bonding and transferring the mirrors to the MHS, the final alignment result was 10.7 arc-seconds RMS diameter, 9.8 arc-seconds HPD.

Table 3. Alignment of pair 489C-2058, September 2010

\begin{tabular}{|l|c|}
\hline Temporary Bond Alignment & $4.0+/-0.3$ arc-seconds \\
\hline Permanent Bond Alignment & $10.7+/-0.3$ arc-seconds \\
\hline
\end{tabular}

All eight of the permanent bonds were less than a micron so the individual point distortion was small. There were some unforeseen issues however such as cutting the epoxy injector syringe tip with a hot blade, and fastening a UV wandholder tower to the base plate. A hot blade was used to cut the syringe tip after bonding, but after four bonds, it was determined that the heat radiating from the tip of the blade was distorting the mirror and it was not used for bonding the second mirror. A new LED UV wand was used for this cycle which required a new tower to hold the wand in place while curing the epoxy. This tower was bolted to the same base plate that the MHS was supported by. It was later revealed that the act of tightening the screw that held the tower down on the base plate to prevent tipping over was distorting the base plate. When the screw was tightened, the whole base plate warped on the sub-micron level, which caused the MHS to tilt slightly, which changed the alignment of the already bonded primary mirror with respect to the secondary mirror that was being bonded.

The previous mirror alignment results for permanent bonded mirrors are produced by optical light Hartmann tests which only rates one type of error seen by the mirror. Performing an x-ray test grazing photons off of the mirrors into a CCD 
detector is the only way of reporting a truly comprehensive result. Since the mirror pair bonded in September 2010 seemed to have a good result, they were taken to x-ray testing. They were put into a four foot diameter vacuum chamber and pumped down to less than $1 \times 10^{\wedge}-5$ Torr at room temperature of $20.4^{\circ} \mathrm{C}$ as shown in Figure 18 . A $4.5 \mathrm{keV}$ titanium source was placed 600 meters downstream from the mirrors and a CCD detector is placed at the 8.4 meter combined focal distance past the mirrors. There is an eight meter section of pipe that connects the chamber to the $\mathrm{x}$-ray $\mathrm{CCD}$.

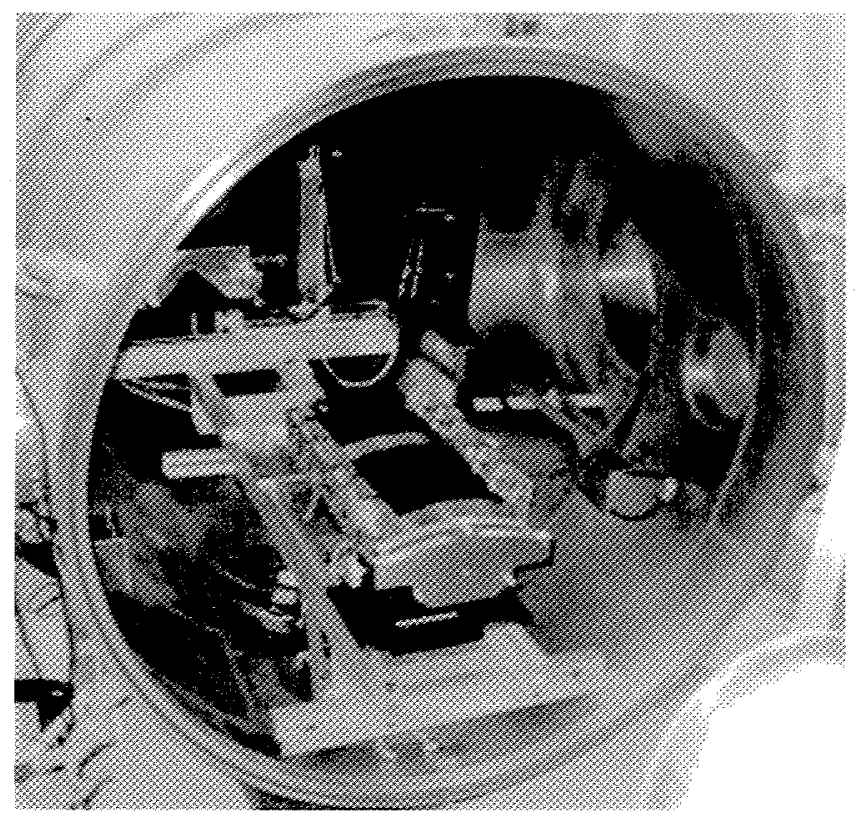

Figure 17 - MHS loaded into vacuum chamber

There were several milestones for the $\mathrm{x}$-ray testing of this mirror pair. This was the first time two mirrors had been permanently bonded to a single fixed structure and x-ray tested. Previously, each mirror was bonded to its own structure and the mirrors could be adjusted relative to each other during the $\mathrm{x}$-ray test to optimize the result. Since both mirrors in the MHS are rigidly attached to a single structure, no further adjustments can be made after the mirrors are permanently bonded.

The x-ray testing on the 489C-2058 mirrors from permanent bond cycle four produced the best results in the history of the IXO project. The best X-ray image was measured at 9.7 arc-seconds HPD for the central 34 degrees of the mirrors. The results are shown in Table 4.

Table 4. X-ray results of mirror pair 489C-2058, October 2010

\begin{tabular}{|c|c|c|}
\hline \multicolumn{3}{|c|}{$\begin{array}{c}\text { X-ray test results } \\
\text { (HPD in arc-seconds) }\end{array}$} \\
\hline Portion of mirror tested & Average & $\begin{array}{c}\text { Standard } \\
\text { Deviation }\end{array}$ \\
\hline $\begin{array}{c}\text { Central 34 degrees } \\
\text { (10 measurements) }\end{array}$ & 11.3 & 1.0 \\
\hline $\begin{array}{c}\text { Full mirror } \\
\text { (5 measurements) }\end{array}$ & 12.7 & 0.9 \\
\hline
\end{tabular}

During the $\mathrm{x}$-ray test, the temperature was cycled from a test temperature of $20.4^{\circ} \mathrm{C}$ up to $27^{\circ} \mathrm{C}$ down to $17^{\circ} \mathrm{C}$, and back to the test temperature. As expected, the HPD result was considerably worse at the higher and lower temperatures. 
The important result of this temperature cycle side-experiment was that the mirror returned to its original shape and the results did not change. The vacuum chamber was also vented to atmospheric pressure halfway through the test. The chamber was opened up and the mirrors were removed from their kinematic mount and then placed back in. After pumping the vacuum chamber back down, the same results were achieved. This helps prove the robustness of the test to outside factors such as temperature, placement inside the chamber, and the pump-down, vent-up processes.

\subsection{Glass 30 degree MHS January permanent bonding and $x$-ray test}

Several bonding runs were made from October to January perfecting the permanent edge bond technique. Figure 18 shows the progress in the resulting alignment of the mirrors after permanent edge bonding. In trial 1, both mirrors were bonded at the same time, no adjustments were made in between mirrors. The six temporary bond edge bond strongback was used (three flexures on top, three screws on bottom). For Trial 2 and onward, the three point temporary edge bond strongbacks had been developed, so only one top flexure and two bottom screws were used. Also for trial 2, a "one mirror at a time" approach was taken. The primary was bonded first. After bonding the primary, the secondary mirror was adjusted on the hexapod to compensate for any distortion caused on the primary mirror component of the mirror pair during bonding. In trial 3, a new type of epoxy was used that was slightly higher viscosity so it didn't "drip" off the end of the pins. For trial 4, since the secondary was already good, only the primary was de-bonded and then re-bonded using the same technique.

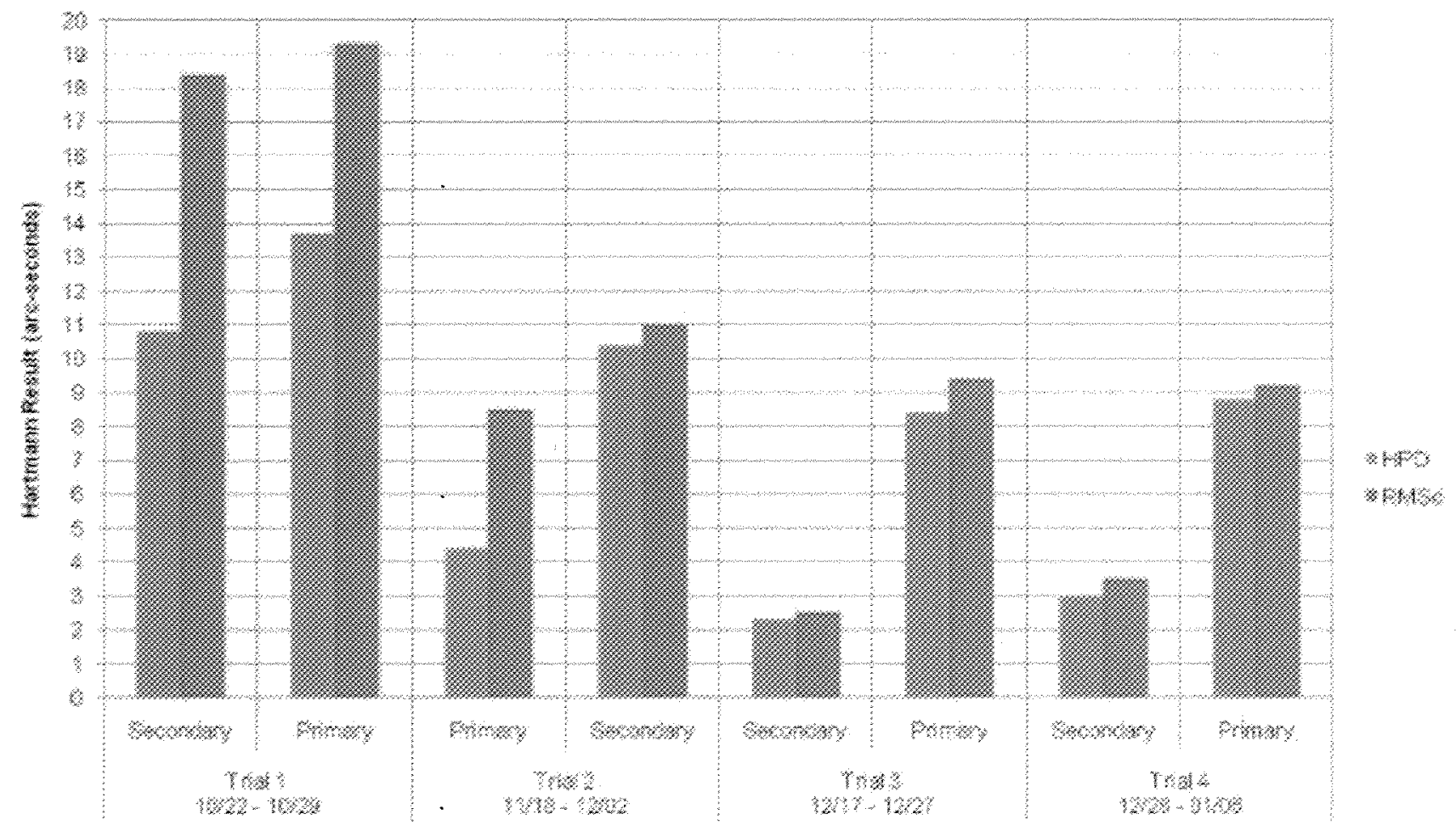

Figure 18 - Edge Bond Pair Progress Summary October to January

Because there was not a significant improvement from trial 3 to trial 4, the permanently bonded mirror pair was taken to $\mathrm{x}$-ray test. A Titanium $4.5 \mathrm{keV}$ x-ray source was used at the NASA GSFC 600 meter beam line. The detector used was $1024 \times 1024$ pixels with a 13 micron pixel size. The mirrors were tested in the "frown" configuration with the GMHS laying on its back in the chamber and the x-ray photons being deflected downwards towards the ground slightly and culminating in a bow-tie shape as shown in Figure 19. 

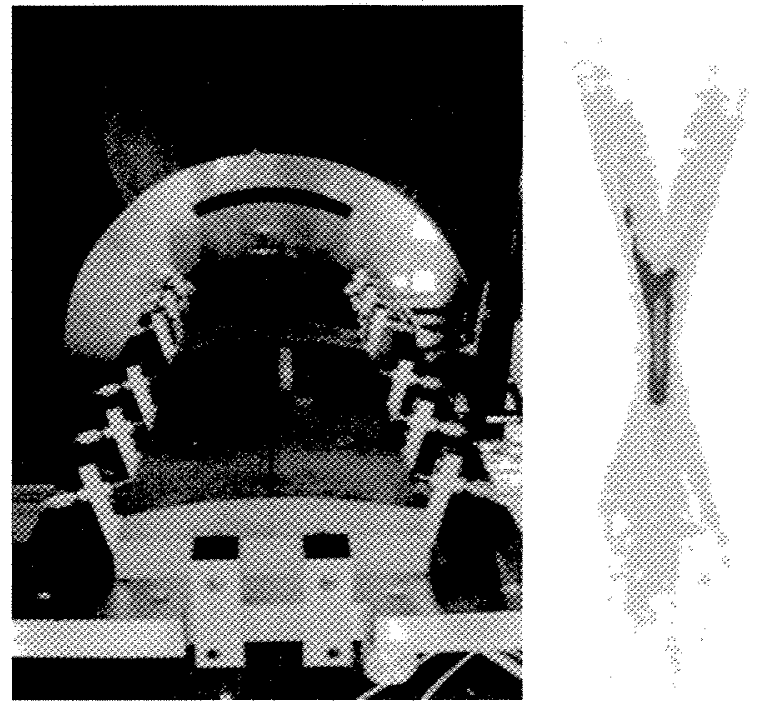

Figure 19 - GMHS in Vacuum Chamber (left), Full Mirror X-Ray Image (right)

Two tests were conducted, one of the full mirror, and a Hartmann test looking at only two degrees of the mirror at a time. For the full mirror image, the results came out to be 9-10 arc-seconds HPD with a best image of 8.9 arc-seconds HPD. The image was acquired using a six minute acquisition time. The Hartmann test showed interesting but expected results of the edges of the mirror being worse than the center. This was to be expected due to the gravity sag of the weight of the mirror since it is laying flat instead of the normal vertical orientation we normally work with. Since our x-ray facility is horizontal (parallel to the ground) this is the way we are constrained to test in. The edges of the mirror were around 15 arc-seconds, and the middle of the mirror was as low as 6.4 arc-seconds. The average across the span of the mirror was 10.04 arc-seconds with an $\mathrm{rms}$ of 2.87 arc-seconds.

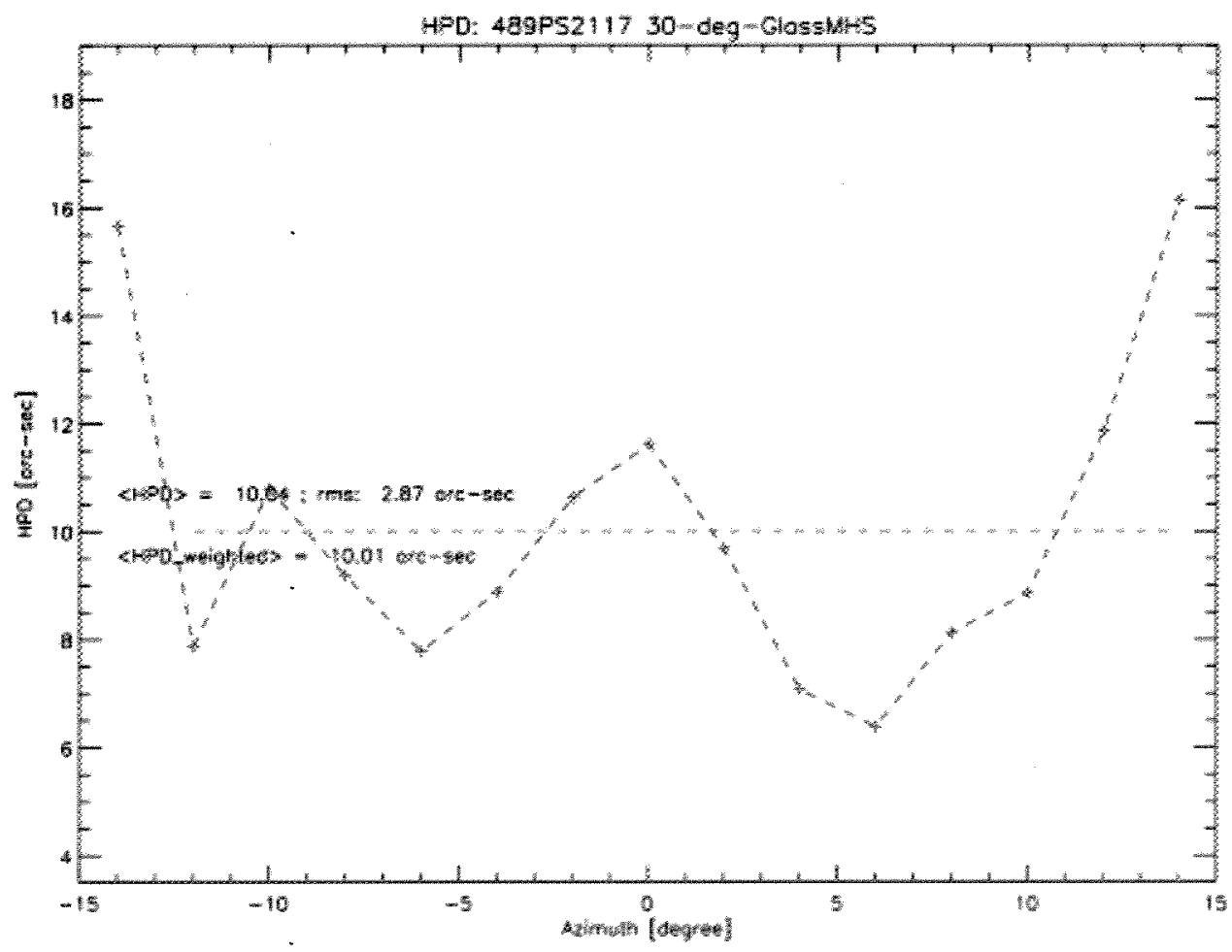

Figure 21 - X-Ray Two Degree Hartmann Test Results in Arc-Seconds HPD 
When plotting the centroids of each centroids for the Hartmann test, the RMSd is 9.2 arc-seconds, which is comparable to the 9.7 arc-seconds measured in alignment after bonding using optical light. The results were very encouraging in that the mirror pair not only came in under the 15 arc-second boundary, but also under the 10 arc-second milestone.

\subsection{Glass 30 Degree MHS March permanent bonding and x-ray test}

Several improvements were made between the January trial and the March trial of the GMHS permanent bonding and xray test. First of all, a new program. was developed for optical light alignment Hartmann tests. The new program named Hartmann Analysis and Diagnostics Software (HADS) not only outputs a centroids plot of the Hartmann test points showing HPD and RMSD, but also prints suggested pitch and yaw correction values. This improved the alignment iteration threshold from two arc-seconds, to one arc-second. In the bonding lab, the ceiling light above the VAF was removed to help stabilize temperature, and the lights were kept off as much as possible. The 3-4 day process was also shortened to two days by performing the Loctite and de-bond step on the same day. This made it possible for only one overnight period of long term stability required to maintain alignment environmentally during the bonding process. The same mirrors were used from the January test after being de-bonded from the GMHS and put back on temporary edge bond strongbacks. The improvements to the procedure helped make this bond trial more successful as can be shown by Figure 22 comparing the January $\mathrm{x}$-ray test to the March $\mathrm{x}$-ray test. In between the two tests, the mirrors were completely de-bonded and then re-aligned, and re-bonded back into the GMHS.

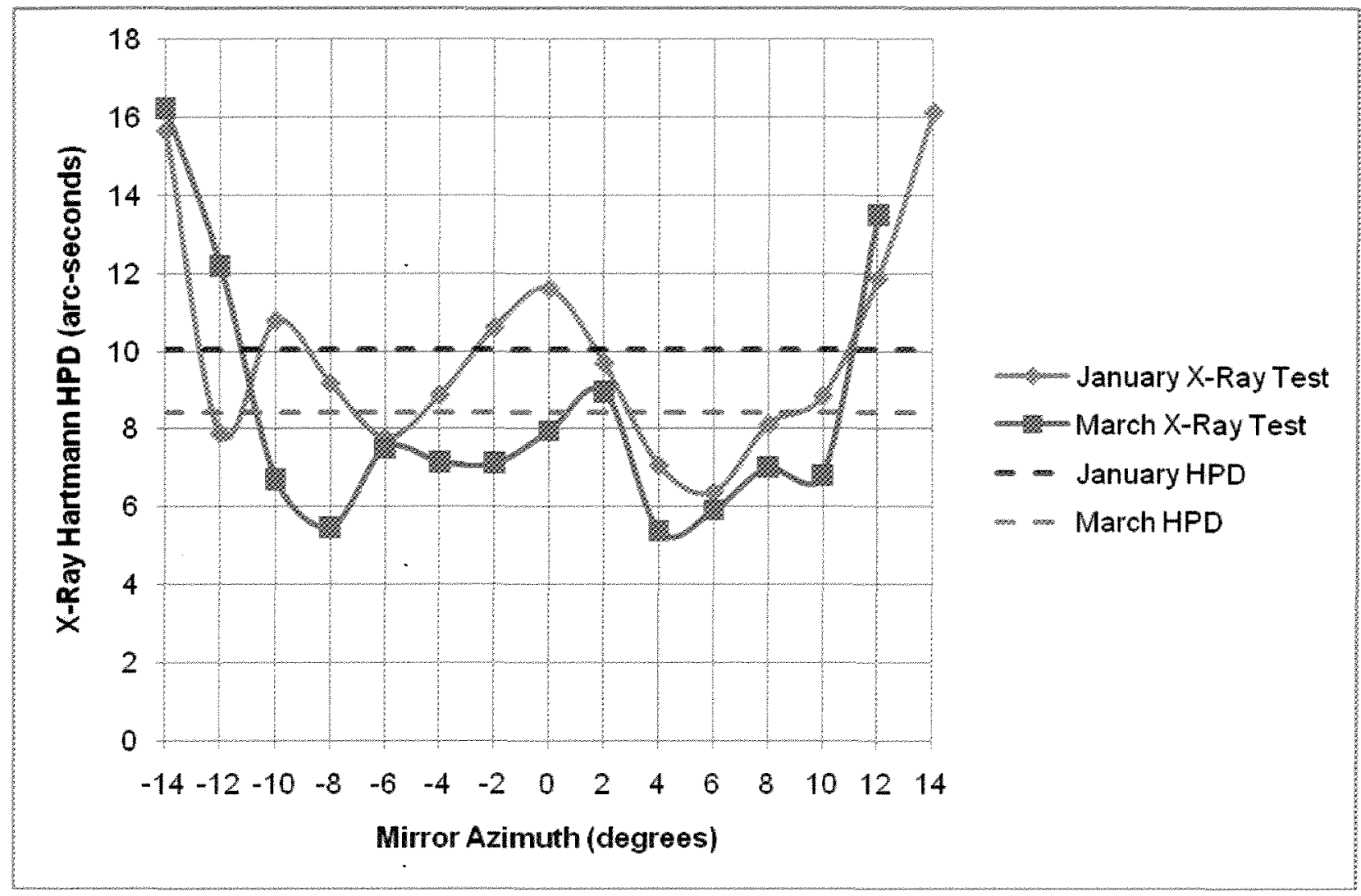

Figure 22 - X-Ray Hartmann HPD Comparison Between January and March Trials

The HPD of the Hartmann test of the different two degree sections of the mirror for the March test ranged from 5.37 arcseconds to 16.23 arc-seconds, again showing better results at the center of the mirror compared to the outer edges. By looking at the comparison data overlaid onto a single graph, it is obvious, that the results from the March trial are better than the January test at almost every point even though the overall HPD average is fairly similar. The substantial take away from this test is that the procedure was repeated successfully with another sub ten arc-second x-ray test. 


\section{CONCLUSIONS}

The mission requirements for IXO of large effective area and high angular resolution do not leave much room for error in the alignment and mounting of thin mirror segments. However, this has driven the design of new hardware and procedures to accommodate these challenges. Now is an exciting time for the alignment and bonding of thin x-ray optics. The improvements and automation of the alignment procedures have helped streamline the effort in improving the alignment iteration threshold resulting in better aligned mirrors for bonding. The automation also helps efficiency, speeding up the entire process which in turn minimizes the time the mirrors are subjected possible environmental changes. It also decreases turn-around time to change procedures and repeat experiments. The method of isolating one variable and repeating an experiment is very valuable in knowing exactly what is being ruled out. If too many variables are changed at once, it is hard to determine what caused the changes in the results. However, this requires many trials when only isolating one variable at a time, so improved cycle time is important. The advances in permanent bonding are exciting in that now mirrors are reliably being permanently bonded into the housing unit while maintaining a sub-ten arc-second alignment. With new procedures and equipment already in place for multiple pair bonding, hopefully the next hurdle of bonding multiple mirror pairs into the housing unit is in the near future to solidify a TRL 4 rating for the technology. With that, environmental testing can commence to qualify the x-ray optics to TRL 5.

\section{REFERENCES}

[1] "The International X-ray Observatory", Nick White, et al., in Proceedings of "X-ray astronomy 2009 Present status, multi-wavelength approach and future perspectives", Astro-Ph, arXiv:1001.2843.

[2] R. McClelland and T. Carnahan, "Design and Analysis of the International X-Ray Observatory Mirror Modules," 2010 IEEE Aerospace Conference Proceedings, March 7-14, 2010.

[3] Zhang, "Lightweight and High Angular Resolution X-ray Optics for Astronomy," Proc. of SPIE Optics and Optoelectronics, Vol. 8076, 2011.

[4] W. Podgorski, D. Caldwell, M. Freeman, et al., "A Mounting and Alignment Approach for Constellation-X Mirror Segments," Proc. of SPIE, 7011, 701112, 2008.

[5] W. Zhang, et al., "IXO Mirror Technology Development," Proc. of SPIE, Vol. 7437, 2009.

[6] R. McClelalnd, T. Carnahan, D. Robinson, T. Saha, "Design and Analysis of the International X-Ray Observatory Mirror Modules" Proc. Of SPIE Vol. 7732, 2010.

[7] K-W. Chan, W. Zhang, T. Saha, et al., "An Approach for Alignment, Mounting and Integration of IXO Mirror Segments," Proc. of SPIE, Vol. 7437, 2009.

[8] T. Saha, S. Rohrbach, W. Zhang, T. Evans, M. Hong, "Grazing incidence wavefront sensing and verification of $x$-ray optics performance" Proc. of SPIE Vol. 8147, 2011.

\section{ACKNOWLEDGEMENTS}

Many people have contributed to the IXO mirror technology development. Thanks go out to Michael Biskach, Peter Blake, Glenn Byron, Tim Carnahan, Kai-Wing Chan, Charles Fleetwood, Melinda Hong, Linette Kolos, Jacob Larimore, Caitlin Lucking, James Mazzarella, Ryan McClelland, Lawrence Olson, Corey Powell, Dave Robinson, Andrew Ross, Timo Saha, Marton Sharpe, David Windle, Kaitlyn Yoha, and Will Zhang. 